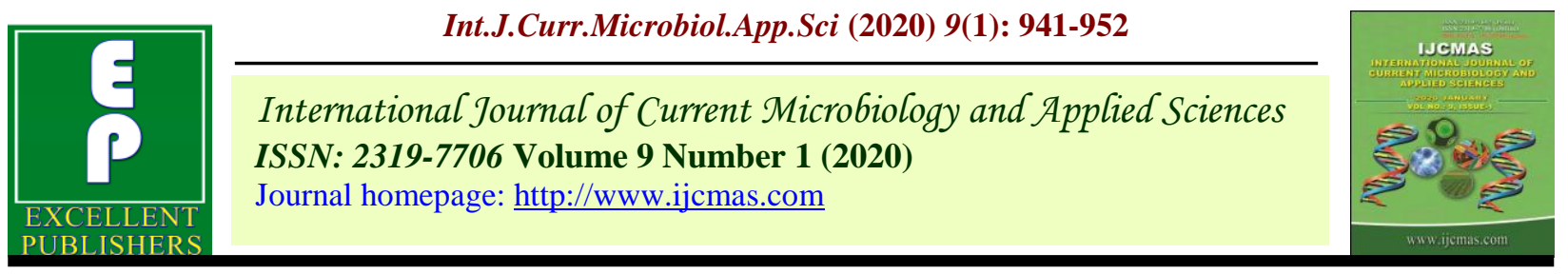

Original Research Article

https://doi.org/10.20546/ijcmas.2020.901.106

\title{
Isolation and Screening of Carotenoid Producing Bacteria
}

\author{
Deepti D. Dhere $^{1 *}$, S. M. Dharmadhikari ${ }^{2}$ and R. S. Pandhare ${ }^{2}$ \\ ${ }^{1}$ Deogiri College of Arts, Science and Commerce, Aurangabad, (M.S.), India \\ ${ }^{2}$ Government College of Arts and Science, Aurangabad, (M.S), India \\ Affiliated to-Dr. Babasaheb Ambedkar Marathwada University, Aurangabad, (M.S.) India \\ *Corresponding author
}

\begin{tabular}{|l|}
\hline K e y w o r d s \\
Screening, DPPH, \\
Carotenoid \\
producing bacteria \\
\hline Article Info \\
\hline $\begin{array}{l}\text { Accepted: } \\
\text { 15 December } 2019 \\
\text { Available Online: } \\
\text { 20 January } 2020\end{array}$ \\
\hline
\end{tabular}

\section{A B S T R A C T}

This article includes isolation and screening of carotenoid producing bacteria. Samples were collected from different eco-climatic regions of Maharashtra. From soil, mud, sediment, and water samples total 620 bacterial strains were isolated out of which 105 isolates were pigment producers, many of which exhibited bright orange, orange, yellow orange, lemon yellow and red pigmentation. Out of 105 isolates efficient carotenoid producers were screened after primary, secondary, tertiary, and quaternary screening based on pigmentation, tolerance to $\mathrm{pH}$ and $\mathrm{NaCl} \%$ (w/v), degree of pigmentation, absorption maxima and free radicle scavenging activity respectively. The selection of potential carotenoid producer was done from finally screened isolatesSL2, SL4*, SL4-1, SL10, SL2y, DKMIII, DABW1, DGM, SSD2, SWT3 and SS1 based on free radicle scavenging activity by DPPH (1,1 Diphenyl-2 Picryl Hydrazil) method. Isolate SL2 showed bright orange pigmentation, tolerance to $\mathrm{pH}(6-12), \mathrm{NaCl}(1-6 \%), \lambda \max$ at $473 \mathrm{~nm}$ and maximum $53 \%$ free radicle scavenging activity, so it was selected for further studies as a potential carotenoid pigment producer.

\section{Introduction}

Natural pigments are always favored by human beings as an additive in food to avoid side effects of the artificial mineral and metalbased colors. The bulk of chemically synthesized colors were derived from aniline and toxic petroleum products. Natural colors are produced mainly by the plants but their availability varies according to season and geographical distribution. Now a day's microbial sources of pigment are emphasized due to their reach biodiversity, year around availability and high production capacity. Carotenoid are C40 compounds which can act as source of pigment as well as therapeutic 
molecule acting as a source of vitamin A, antioxidants or possible tumor inhibiting agent. Carotenoids pigments are present in all photosynthetic organisms as well as in some photosynthetic and non-photosynthetic bacteria, fungi, yeast, and algae. The nonphotosynthetic microorganisms produce carotenoids where, carotenoids protect cell from stressed environment by altering membrane fluidity. Bacteria are more convenient for large scale production of carotenoid than algae and fungi due to their unicellular nature, relatively high growth rate, easy handling, and processing. This research article describes the isolation and screening of carotenoid producing bacteria from different environmental niche and selection of potential carotenoid producer for further studies. For isolation of carotenoid producing bacteria selective enrichment culture technique was used to favor the multiplication of bacteria from the sample which were low in number initially.

\section{Materials and Methods}

\section{Sample collection and enrichment}

For isolation of carotenoid producing bacteria, random sampling was done in the month of May and June. Different samples (water, soil, mud, and sediment) were collected in sterile container and stored at $4^{\circ} \mathrm{C}$ prior to use.

The samples collected includes hypersalinehyperalkaline environment of Lonar crater, Buldhana, Maharashtra, Marine water from Kerala, Goa, and Mumbai sea shore. Some samples were also collected from different regions of Aurangabad such as water samples from Panchakki; soil, mud, sediment, and water samples from Harsul lake and Dr. Salim Ali Lake, Aurangabad and Hatala lake, Hingoli. Enrichment of samples was done in Nutrient broth and incubated for $48 \mathrm{~h}$ in presence of day light at room temperature.

\section{Isolation and primary Screening}

Isolation was done by serial dilution of enriched culture in physiological saline $(0.85 \% \mathrm{NaCl})$ and $0.1 \mathrm{~mL}$ of each diluent was used for spreading the nutrient agar $(\mathrm{pH} 8.5)$ with $1.5 \%$ agar-agar. The plates were prepared in duplicates and incubated for $48 \mathrm{~h}$ in day light near window at room temperature. After incubation, plates with isolated colonies with pigmentation were selected as master plates and kept for further use. Primary screening of all pigment producing cultures was carried out by growing pigmented isolates from master plates on nutrient agar with $\mathrm{pH} 8.5$ at room temperature for $48 \mathrm{~h}$ in duplicates. The primary screening of isolates was done based on ability to produce yellow, orange or red pigment which likely to be non-diffusible among other pigment producers and nonpigmented cultures on plates. The isolates showing yellow, orange and red pigmentation were separated and transferred on nutrient agar slants and preserved at $4^{0} \mathrm{C}$ for further studies.

\section{Secondary screening}

The Secondary screening was done based on qualitative tolerance to $\mathrm{pH}$ and salt $(\mathrm{NaCl} \%)$. All primarily screened bacterial isolates were streaked on nutrient agar with different $\mathrm{pH}$ from 5-12 and nutrient agar consisting $\mathrm{NaCl}$ (1-6\%) separately. The plates were incubated at room temperature in day light near window for $48 \mathrm{~h}$.

The isolates growing on nutrient agar with wide range of $\mathrm{pH}$ were considered as tolerant cultures and streaked on nutrient agar with higher $\mathrm{NaCl}$ i.e. 1-6\%. Cultures showing good pigmentation with $\mathrm{pH}$ and $\mathrm{NaCl}$ tolerance while retaining its bright pigmentation were screened secondarily and selected for tertiary screening. 


\section{Tertiary screening}

Tertiary screening was done based on qualitative and quantitative performance of the isolate to produce pigment. In tertiary screening, actively growing ( $48 \mathrm{~h}$ ) cultures of secondarily screened isolates were inoculated separately into nutrient broth ( $\mathrm{pH} 8.5$ and $1 \%$ $\mathrm{NaCl}$ ) and incubated with shaking condition (100 rpm) at room temperature for $48 \mathrm{~h}$. The culture with vigorous growth along with bright pigmentation were subjected to extraction of pigment by slight modification of the procedure used by Bhat et al., (2015) where, the enriched cultures were centrifuged separately at $8000 \mathrm{rpm}$ for $15 \mathrm{~min}$. at $4^{0} \mathrm{C}$ to separate cells. Separated cell pellets were washed twice with sterile distilled water by centrifugation at $8000 \mathrm{rpm}$ for $10 \mathrm{~min}$. The cell pellets were suspended separately in 5 $\mathrm{mL}$ of methanol and kept overnight, followed by centrifugation at $8000 \mathrm{rpm}$ for $10 \mathrm{~min}$. at $4^{0} \mathrm{C}$. The supernatants were separately analyzed spectrophotometrically between 400-600 $\mathrm{nm}$ to find the $\lambda_{\max }$. During tertiary screening degree of pigmentation was also calculated. Degree of pigmentation is the ratio of pigment produced to the biomass produced where, ratio of absorbance of the pigmented extract at its respective $\lambda \max$ to the absorbance of $48 \mathrm{~h}$ culture at $660 \mathrm{~nm}$ was calculated. The isolate showing higher values for degree of pigmentation and characteristic $\lambda_{\max }$ of carotenoids were selected for quaternary screening.

\section{Quaternary screening}

Quaternary screening was done based antioxidant activity and hence it was done by evaluating antioxidant potential of pigment extract from tertiary screened isolates. The antioxidant potential was estimated by evaluating free radical scavenging activity as per procedure given by Sasidharan et al., (2013) with slight modifications where, 1,1diphenyl-2-picryl-hydrazyl (DPPH) was used.
The solution of DPPH $0.1 \mathrm{mM}$ was prepared in methanol and of this $1 \mathrm{~mL}$ was added $2 \mathrm{~mL}$ of pigment extract in methanol. The mixture was shaken vigorously and allowed to stand at room temperature for $30 \mathrm{~min}$. The whole steps of DPPH assay were carried out in subdued light. After incubation absorbance of different carotenoid extracts under assay were measured separately at $517 \mathrm{~nm}$ by using UVvisible spectrophotometer. The percentage of DPPH scavenging activity was calculated by using the equation. The control reaction was carried out by taking $1 \mathrm{~mL}$ of $0.1 \mathrm{mM} \mathrm{DPPH}$ solution and $2 \mathrm{~mL}$ of methanol with same treatment as to test samples. DPPH is stable radical and in solution it is dark violet in color and it is decolorized by potent reducing substances and antioxidants. High rate of decolorization with pigment extract suggest better antioxidant activity. The extract from bacterial isolate showing highest free radical scavenging activity i.e. \% RSA was considered as potential antioxidant function and isolate as potential carotenoid producer. The radical scavenging activity (\% RSA) of the methanol fraction of carotenoids extracts were measured and calculated by-

$\% \mathrm{RSA}=\left(1-\frac{\text { absorbance of sample }}{\text { absorbanceof control }}\right) \times 100$

The color of bacterial cell pellet was matched by Munsell color chart by using Munsell color code Android app as 'DIC Color Guide'as per Kollmorgen Corporation, "Munsell color charts for plant tissue" given by Munsell Color division, Maryland (1972).

\section{Results and Discussion}

\section{Sample collection and enrichment}

The sampling was done by considering biodiversity and uniqueness of source from which samples were collected. Samples were collected from different locations of Maharashtra state and India (Table-1a) 
(Figure-1a, 1b). Total 88 samples i.e. soil, mud, sediment, and water were collected and subjected for enrichment in nutrient broth $\mathrm{pH}$ 8.5 where, water samples were analyzed for their physiological parameters before using as an inoculum for enrichment (Table 1b). Maximum number of samples i.e. $31 \%$ was collected from Lonar crater while least number of samples was collected from fresh water lakes. Zeni et al., (2011) collected soil, leaves (Eucalyptus and orange), fruits (orange, papaya, persimmon, peach, apple etc.) teas, and agro-industrial wastes for isolation of carotenoid producing microorganisms while, Brown, (1963) used sediment samples for isolation of carotenoid producing bacteria. Arunkumar et al., (2006) and Shatila et al., (2013) done air sampling for isolation of carotenoid producing bacteria. Godlinho et al., (2008) isolated orange pigmented bacteria from rhizosphere of sand dune creeper-Ipomoea pes-caprae while Arulselvi et al., (2014) used soil samples for isolation of yellow pigment producing bacteria. Pathak et al., (2012) and Oren et al., (2001) used brine samples for isolation of carotenoid producing haloarchaea and halophilic bacteria respectively. Sasidharan et al., (2013) isolated $67 \%$ pigmented isolates from soil and $33 \%$ from air. In the present studies the variety of samples were used which includes soil, water (marine, hypersaline-hyperalkaline, and fresh water), mud and sediment of these ecosystems for isolation of carotenoid pigment producing bacteria. There are very few studies reported where, similar samples were collected and studied combinedly for isolation of carotenoid producing bacteria. From the data generated from current research (Table-1a and 1b), from 88 samples, 105 yellow, orange, and red pigment producing bacterial isolates were recovered. Therefore, it can be assumed that growth carotenoid producing bacteria can be induced by extreme environments as carotenoids are produced by bacteria in response to extreme environment and adverse growth conditions.

\section{Isolation and primary screening}

The isolation of carotenoid producing bacteria was done from enriched samples by serial dilution and spread plate technique on nutrient agar $\mathrm{pH}$ 8.5. After incubation, total 620 bacterial isolates were recovered. Out of 620 bacterial isolates 515 isolates were not having any type of pigment and 105 isolates were with yellow, orange, yellow orange, lemon yellow and red pigmentation which were subjected for screening.

Carotenoids are pigments with yellow, orange and red pigmentation hence, all isolates producing bright yellow, yellow orange, lemon yellow, orange and red pigment on nutrient agar were screened primarily among pigmented and non-pigmented isolates. Table$2 \mathrm{a}, 2 \mathrm{~b}$ and figure-2a, $2 \mathrm{~b}$ shows the distribution of different pigment producing bacteria among isolates. Highest number of isolates was recovered with yellow orange and lemon-yellow pigmentation i.e. $32 \%$, and $29 \%$ respectively while, least quantity of red pigmented isolate was recovered i.e. $4 \%$. Bright orange and orange pigmentation was found with the $11 \%$ and $24 \%$ isolates respectively. Godinho and Bhosle, (2008) isolated orange pigment producing alkaliphilic bacterial strain Microbacterium arborescens-AGBS from rhizosphere of costal sand dune creeper while, Balraj et al., (2014)isolated yellow pigmented marine bacteria from sea water of Peninsular Region of India. They have isolated 61 different bacterial strains out of which $19(31 \%)$ strains were pigmented and out of 19 pigmented strains $11(58 \%)$ were yellow and $6(32 \%)$ were orange pigment producing strains and remaining $2(10 \%)$ strains were peach in color. The probabilities of pigment producing bacteria from this study are similar with 
present research findings where maximum yellow pigmented bacterial isolates were recovered. None of reports were found on isolation and screening of carotenoid producing bacteria from hypersalinehyperalkaline environment so it is the first report on isolation and screening of carotenoid producing bacteria from such said extreme environment. The present research finding also highlights that samples from extreme environment are rich source of pigment producing bacteria.

Table.1a Distribution of samples collected

\begin{tabular}{|c|c|c|c|c|c|c|c|}
\hline $\begin{array}{c}\text { Sr. } \\
\text { No. }\end{array}$ & Sample source & $\begin{array}{c}\text { Geographical } \\
\text { Location }\end{array}$ & Soil & Mud & Water & Sediment & Total \\
\hline $\mathbf{1}$ & Lonar Crater, Buldhana & $\begin{array}{c}19.9758^{\circ} \mathrm{N}, \\
76.5069^{\circ} \mathrm{E}\end{array}$ & 18 & 05 & 13 & 07 & 43 \\
\hline $\mathbf{2}$ & Marine water sample & -- & -- & -- & 23 & -- & 23 \\
\hline $\mathbf{3}$ & Dr. Salim Ali lake, Aurangabad & $19.8992^{\circ} \mathrm{N}$, & 05 & -- & 03 & 05 & 13 \\
\hline $\mathbf{4}$ & Harsul Lake, Aurangabad & $\begin{array}{c}75.3423^{\circ} \mathrm{E} \\
19.9282^{\circ} \mathrm{N},\end{array}$ & 01 & -- & 05 & 08 & 14 \\
\hline $\mathbf{5}$ & Panchakki, Aurangabad & $\begin{array}{c}75.3368^{\circ} \mathrm{E} \\
19.8896^{\circ} \mathrm{N},\end{array}$ & -- & -- & 05 & -- & 05 \\
\hline $\mathbf{6}$ & Hatala Lake, Hingoli & $\begin{array}{c}75.3155^{\circ} \mathrm{E} \\
77.174^{\circ} \mathrm{N},\end{array}$ & -- & -- & 07 & -- & 07 \\
\hline & & Total & 24 & 05 & 56 & 20 & 105 \\
\hline
\end{tabular}

Table.1b Physicochemical properties of the sample collected

\begin{tabular}{|l|l|c|c|c|c|c|}
\hline $\begin{array}{c}\text { Sr. } \\
\text { No. }\end{array}$ & \multicolumn{1}{|c|}{ Sample source } & pH & $\begin{array}{c}\text { Temperature } \\
\left(\mathbf{(}^{\mathbf{C}}\right)\end{array}$ & Turbidity & Odor & Color \\
\hline $\mathbf{1}$ & Lonar Crater, Buldhana & $10.8 \pm 0.2$ & 36 & $\mathrm{P}$ & Algal & Colorless \\
\hline $\mathbf{2}$ & Marine sample & $7.2 \pm 0.2$ & 35 & NP & NP & Colorless \\
\hline $\mathbf{3}$ & $\begin{array}{l}\text { Dr. Salim Ali lake, } \\
\text { Aurangabad }\end{array}$ & $8.8 \pm 0.2$ & 32 & $\mathrm{P}$ & Pungent & Turbid \\
\hline $\mathbf{4}$ & Harsul Lake, Aurangabad & $7.8 \pm 0.2$ & 32 & $\mathrm{NP}$ & $\mathrm{NP}$ & Colorless \\
\hline $\mathbf{5}$ & Panchakki, Aurangabad & $7.4 \pm 0.2$ & 30 & $\mathrm{NP}$ & Algal & Colorless \\
\hline $\mathbf{6}$ & Hatala Lake, Hingoli & $7.2 \pm 0.2$ & 30 & NP & Algal & Colorless \\
\hline
\end{tabular}

Table.2a Distribution of isolates according to sample source and pigmentation

\begin{tabular}{|c|c|c|c|c|c|c|c|c|}
\hline $\begin{array}{c}\text { Sr. } \\
\text { No. }\end{array}$ & $\begin{array}{c}\text { Pigmentation } \\
\text { Sample source }\end{array}$ & $\begin{array}{c}\text { Non- } \\
\text { pigmented } \\
\text { isolates }\end{array}$ & $\begin{array}{c}\text { Bright } \\
\text { Orange }\end{array}$ & Orange & $\begin{array}{c}\text { Yellow } \\
\text { Orange }\end{array}$ & $\begin{array}{c}\text { Lemon } \\
\text { Yellow }\end{array}$ & Red & Total \\
\hline $\mathbf{1}$ & $\begin{array}{c}\text { Lonar Crater, } \\
\text { Buldhana. }\end{array}$ & 103 & 09 & 13 & 10 & 09 & 02 & 146 \\
\hline $\mathbf{2}$ & Marine water samples. & 96 & 01 & 04 & 06 & 12 & 0 & 119 \\
\hline $\mathbf{3}$ & Dr. Salim Ali lake samples. & 112 & 01 & 01 & 07 & 02 & 02 & 125 \\
\hline $\mathbf{4}$ & Harsul Lake water. & 87 & 00 & 05 & 06 & 03 & 00 & 101 \\
\hline $\mathbf{5}$ & Panchakki water. & 49 & 01 & 02 & 02 & 00 & 00 & 54 \\
\hline $\mathbf{6}$ & Hatala Lake water & 68 & 00 & 00 & 03 & 04 & 00 & 75 \\
\hline & Total & 515 & 12 & 25 & 34 & 30 & 04 & 620 \\
\hline
\end{tabular}


Table.2b Distribution of pigmented isolates according to ecosystem selected

\begin{tabular}{|c|c|c|c|}
\hline Pigment produced & \multicolumn{3}{|c|}{ Number of isolates from ecosystem selected } \\
\cline { 2 - 4 } & Lonar Crater & Marine Water & Fresh water \\
\hline Bright orange & 08 & 01 & 03 \\
\hline Orange & 14 & 04 & 08 \\
\hline Yellow orange & 10 & 06 & 19 \\
\hline Lemon yellow & 09 & 12 & 07 \\
\hline Red & 02 & 00 & 02 \\
\hline Total & 43 & 23 & 40 \\
\hline
\end{tabular}

Table.3a Secondary screening of carotenoid producing bacteria from Lonar Crater

\begin{tabular}{|c|c|c|c|c|c|c|c|c|c|c|c|}
\hline \multirow{2}{*}{$\begin{array}{l}\text { Sr. } \\
\text { No. }\end{array}$} & \multirow{2}{*}{$\begin{array}{l}\text { Name of } \\
\text { Culture }\end{array}$} & \multirow[t]{2}{*}{ Pigment } & \multirow{2}{*}{$\begin{array}{c}\mathrm{pH} \\
\text { tolerance }\end{array}$} & \multirow{2}{*}{$\begin{array}{l}\mathrm{NaCl}(\%) \\
\text { tolerance }\end{array}$} & \multicolumn{7}{|c|}{ Variation in pigment $(\mathrm{pH})$} \\
\hline & & & & & 6 & 7 & 8.5 & 9.5 & 10.5 & 11 & 12 \\
\hline $\mathbf{1}$ & SL2 & Bright orange & $6-12$ & $1-6$ & $\mathbf{O}$ & BO & BO & BO & BO & BO & BO \\
\hline 2 & SL2** & Orange & $7-12$ & $1-6$ & W & FO & $\mathrm{O}$ & $\mathrm{O}$ & $\mathrm{O}$ & $\mathrm{O}$ & $\mathrm{P}$ \\
\hline 3 & SL4-1 & Yellow orange & $6-12$ & $1-5$ & $\mathbf{O}$ & YO & YO & YO & YO & $\mathbf{O}$ & $\mathbf{O}$ \\
\hline 4 & SD4 & Bright orange & $6-12$ & $1-3$ & $\mathrm{BO}$ & LO & LO & LO & LO & LO & LO \\
\hline 5 & SL9 & Orange & $6-12$ & $1-6$ & $\mathbf{O}$ & $\mathbf{O}$ & YO & BO & BO & BO & BO \\
\hline 6 & SL10 & Bright orange & $6-12$ & $1-3$ & LO & BO & BO & BO & BO & BO & BO \\
\hline 7 & IC & Dark Red & $5-12$ & $1-4$ & DR & DR & DR & DR & DR & DR & DR \\
\hline 8 & LBSL4 & Yellow orange & $6-12$ & $1-4$ & $\mathrm{O}$ & $\mathrm{FO}$ & FO & $\mathrm{FO}$ & $\mathrm{FO}$ & $\mathrm{FO}$ & $\mathrm{FO}$ \\
\hline 9 & SL2y & Lemon yellow & $6-12$ & $1-5$ & FY & LY & LY & LY & LY & LY & LY \\
\hline 10 & LWT1 & Orange & $6-12$ & $1-4$ & $\mathrm{O}$ & $\mathrm{P}$ & FO & $\mathrm{FO}$ & FO & $\mathrm{P}$ & $\mathrm{P}$ \\
\hline 11 & SL6 & Orange & $6-12$ & $1-4$ & $\mathrm{O}$ & $\mathrm{BO}$ & YO & YO & YO & $\mathrm{O}$ & $\mathrm{O}$ \\
\hline 12 & SL4* & Orange & $6-12$ & $1-6$ & FO & $\mathbf{O}$ & $\mathbf{O}$ & FO & FO & $\mathbf{P}$ & $\mathbf{P}$ \\
\hline
\end{tabular}

Table.3b Secondary screening of carotenoid producing bacteria from marine samples

\begin{tabular}{|l|l|l|l|l|l|l|l|l|l|l|l|}
\hline $\begin{array}{l}\text { Sr.N } \\
\text { o. }\end{array}$ & $\begin{array}{l}\text { Name of } \\
\text { Culture }\end{array}$ & Pigment & $\begin{array}{l}\text { pH } \\
\text { tolerance }\end{array}$ & $\begin{array}{l}\text { NaCl } \\
\text { tolerance }\end{array}$ & $\mathbf{6}$ & $\mathbf{7}$ & $\mathbf{8 . 5}$ & $\mathbf{9 . 5}$ & $\mathbf{1 0 . 5}$ & $\mathbf{1 1}$ & $\mathbf{1 2}$ \\
\hline $\mathbf{1}$ & AN9 & Lemon Yellow & $\mathbf{6 - 8}$ & $\mathbf{3 - 6}$ & LY & LY & LY & NG & NG & NG & NG \\
\hline $\mathbf{2}$ & ANN10 & Orange & $\mathbf{6 - 1 0}$ & $\mathbf{3 - 6}$ & $\mathbf{O}$ & $\mathbf{O}$ & $\mathbf{O}$ & $\mathbf{O}$ & $\mathbf{O}$ & FO & FO \\
\hline $\mathbf{3}$ & DKMO & Yellow orange & $5-10$ & $3-5$ & YO & YO & YO & YO & YO & NG & NG \\
\hline $\mathbf{4}$ & DKMIII & Yellow orange & $\mathbf{6 - 8}$ & $\mathbf{3 - 4}$ & YO & YO & YO & FO & NG & NG & NG \\
\hline $\mathbf{5}$ & ANAB5 & Bright orange & $\mathbf{6 - 8}$ & $3-6$ & BO & BO & BO & FO & FO & NG & NG \\
\hline $\mathbf{6}$ & DABW1 & Orange & $\mathbf{6 - 8}$ & $\mathbf{7 3}$ & $\mathbf{O}$ & $\mathbf{O}$ & $\mathbf{O}$ & FO & FO & P & NG \\
\hline $\mathbf{7}$ & DGM & Lemon yellow & $\mathbf{6 - 1 0}$ & $\mathbf{3 - 8}$ & LY & LY & LY & LY & LY & NG & NG \\
\hline $\mathbf{8}$ & ANG7 & Lemon yellow & $6-10$ & $3-6$ & LY & LY & LY & LY & LY & NG & NG \\
\hline $\mathbf{9}$ & ANB6 & Lemon yellow & $\mathbf{6 - 1 0}$ & $\mathbf{3 - 6}$ & LY & LY & LY & LY & LY & W & W \\
\hline $\mathbf{1 0}$ & ANB11 & Lemon yellow & $6-10$ & $3-6$ & LY & LY & LY & LY & LY & NG & NG \\
\hline $\mathbf{1 1}$ & DKMY & Yellow orange & $\mathbf{6 - 1 0}$ & $\mathbf{3 - 5}$ & YO & YO & YO & YO & FO & W & NG \\
\hline $\mathbf{1 2}$ & DKN11 & Yellow orange & $\mathbf{6 - 8}$ & $\mathbf{3 - 5}$ & YO & YO & YO & FO & FO & W & NG \\
\hline
\end{tabular}


Table.3c Secondary screening of carotenoid producing bacteria from Aurangabad and Hingoli samples

\begin{tabular}{|c|c|c|c|c|c|c|c|c|c|c|c|c|}
\hline $\begin{array}{c}\text { Sr. } \\
\text { No. }\end{array}$ & $\begin{array}{l}\text { Name of } \\
\text { Culture }\end{array}$ & Pigment & $\begin{array}{c}\text { pH } \\
\text { tolerance }\end{array}$ & $\begin{array}{c}\text { NaCl (\%) } \\
\text { tolerance }\end{array}$ & \multicolumn{4}{|c|}{ Variation in pigment (pH) } \\
\hline $\mathbf{1}$ & SSD1 & Yellow orange & $5-11$ & $1-4$ & YO & YO & YO & YO & YO & FY & NG & NG \\
\hline $\mathbf{2}$ & SSD2 & Orange & $\mathbf{5 - 1 1}$ & $\mathbf{1 - 3}$ & $\mathbf{O}$ & $\mathbf{O}$ & $\mathbf{O}$ & $\mathbf{O}$ & FO & FO & P & P \\
\hline $\mathbf{3}$ & SSD4 & Yellow orange & $5-11$ & 1 & YO & YO & YO & FO & FO & FO & FO & NG \\
\hline $\mathbf{4}$ & SWT2 & Yellow orange & $5-11$ & $1-4$ & YO & YO & YO & FO & FO & FO & FO & NG \\
\hline $\mathbf{5}$ & SWT3 & Red & $\mathbf{5 - 1 1}$ & $\mathbf{1 - 2}$ & DR & DR & DR & DR & DR & DR & DR & NG \\
\hline $\mathbf{6}$ & SS1 & Bright orange & $\mathbf{5 - 1 1}$ & $\mathbf{2 - 3}$ & $\mathbf{O}$ & $\mathbf{O}$ & $\mathbf{B O}$ & $\mathbf{B O}$ & $\mathbf{B O}$ & $\mathbf{O}$ & $\mathbf{O}$ & $\mathbf{P}$ \\
\hline $\mathbf{7}$ & HSD6 & Yellow orange & $5-10$ & $1-4$ & FY & FY & YO & YO & YO & FY & NG & NG \\
\hline
\end{tabular}

Legend for table-3a., 3b. and 3c.YO- yellow orange, FY- faint yellow, NG- no growth, FO- faint orange, DR-dark red, BO-bright orange, O-orange, W-white, FP- faint pink, P- pink, LY-lemon yellow and LO-light orange.

Table.4a Tertiary screening of bacteria from Lonar Crater based on extraction and characterization along with degree of pigmentation

\begin{tabular}{|c|c|c|c|c|c|c|c|}
\hline $\begin{array}{l}\text { Sr. } \\
\text { No. }\end{array}$ & $\begin{array}{l}\text { Bacterial } \\
\text { culture }\end{array}$ & $\begin{array}{c}\text { Wet weight } \\
\text { of } \\
\text { pellet } / 100 \mathrm{ml}\end{array}$ & $\begin{array}{c}\text { Absorption } \\
\text { maxima }(\lambda \max ) \\
(\mathbf{n m})\end{array}$ & $\begin{array}{c}\text { O.D.at } \\
\lambda \max (\mathbf{n m})\end{array}$ & $\begin{array}{l}\text { O.D. at } \\
\text { 660nm }\end{array}$ & DOP & $\begin{array}{c}\text { Predicted } \\
\text { pigment }\end{array}$ \\
\hline 1 & SL2 & 0.941 & 473 & 3.796 & 1.350 & 2.81 & Astaxanthin \\
\hline 2 & SL2** & 0.534 & 466 & 0.272 & 0.968 & 0.28 & Cryptoxanthin \\
\hline 3 & SL4-1 & 0.773 & 443 & 2.220 & 1.446 & 1.53 & Zeaxanthin \\
\hline 4 & SD4 & 0.528 & 446 & 0.215 & 1.235 & 0.17 & Zeaxanthin \\
\hline 5 & SL9 & 1.067 & 475 & 1.345 & 1.363 & 0.98 & Astaxanthin \\
\hline 6 & SL10 & 0.699 & 475 & 1.620 & 1.162 & 1.39 & Astaxanthin \\
\hline 7 & IC & 0.542 & 536 & 2.54 & 0.391 & 6.41 & Prodigiosin \\
\hline 8 & LBSL4 & 0.619 & 411 & 0.390 & 1.167 & 0.33 & -- \\
\hline 9 & SL2y & 1.361 & 437 & 2.868 & 1.993 & 1.43 & Cryptoxanthin \\
\hline 10 & LWT1 & 0.542 & 449 & 0.266 & 0.779 & 0.34 & $\beta$-Carotene \\
\hline 11 & SL6 & 0.873 & 440 & 1.022 & 1.655 & 0.61 & Zeaxanthin \\
\hline 12 & SL4* & 1.100 & 449 & 1.576 & 1.829 & 0.86 & $\beta$-Carotene \\
\hline
\end{tabular}

Table.4b Tertiary screening of bacteria from marine samples based on extraction and characterization along with degree of pigmentation

\begin{tabular}{|c|c|c|c|c|c|c|c|}
\hline $\begin{array}{l}\text { Sr. } \\
\text { No }\end{array}$ & $\begin{array}{l}\text { Bacterial } \\
\text { culture }\end{array}$ & $\begin{array}{c}\text { Wet weight of } \\
\text { pellet } / 100 \mathrm{ml}\end{array}$ & $\begin{array}{c}\text { Absorption maxima } \\
(\lambda \max -\mathrm{nm})\end{array}$ & $\begin{array}{c}\text { O.D. at } \\
\lambda \max -n m\end{array}$ & $\begin{array}{l}\text { O.D. at } \\
\text { 660nm }\end{array}$ & DOP & $\begin{array}{c}\text { Predicted } \\
\text { pigment }\end{array}$ \\
\hline 1 & AN9 & 0.175 & 437 & 0.474 & 1.375 & 0.34 & Cryptoxanthin \\
\hline 2 & DKN11 & 0.940 & 437 & 0.598 & 1.777 & 0.33 & Cryptoxanthin \\
\hline 3 & DKMIII & 0.237 & 450 & 1.354 & 1.347 & 1.00 & $\beta$-Carotene \\
\hline 4 & DABW1 & 0.234 & 463 & 0.912 & 1.363 & 0.66 & Cryptoxanthin \\
\hline 5 & DGM & 0.232 & 436 & 0.702 & 1.706 & 0.41 & Cryptoxanthin \\
\hline 6 & ANB6 & 0.423 & 438 & 0.281 & 1.880 & 0.14 & Cryptoxanthin \\
\hline 7 & DKMY & 0.884 & 436 & 0.580 & 1.817 & 0.30 & Cryptoxanthin \\
\hline
\end{tabular}


Table.4c Tertiary screening of bacteria from Aurangabad and Hingoli samples based on extraction and characterization along with degree of pigmentation

\begin{tabular}{|c|c|c|c|c|c|c|c|}
\hline $\begin{array}{c}\text { Sr. } \\
\text { No. }\end{array}$ & $\begin{array}{c}\text { Bacterial } \\
\text { culture }\end{array}$ & $\begin{array}{c}\text { Wet weight of } \\
\text { pellet/100ml }\end{array}$ & $\begin{array}{c}\text { Absorption maxima } \\
(\mathbf{\lambda} \mathbf{m a x}-\mathbf{n m})\end{array}$ & $\begin{array}{c}\text { OD at } \\
\text { (max-(nm) }\end{array}$ & O.D. 660nm & DOP & $\begin{array}{c}\text { Predicted } \\
\text { pigment }\end{array}$ \\
\hline $\mathbf{1}$ & SSD2 & 0.347 & 468 & 0.771 & 0.834 & 0.93 & Canthaxanthin \\
\hline $\mathbf{2}$ & SWT3 & 0.921 & 536 & 4.63 & 1.723 & 2.69 & Prodigiosin \\
\hline $\mathbf{3}$ & SS1 & $\mathbf{0 . 5 3 2}$ & $\mathbf{4 7 4}$ & $\mathbf{1 . 5 4}$ & $\mathbf{1 . 0 8 9}$ & $\mathbf{1 . 4 2}$ & Astaxanthin \\
\hline
\end{tabular}

Table.5a Quaternary screening of carotenoid producing bacteria isolated from Lonar Crater

\begin{tabular}{|c|c|c|c|c|c|c|c|}
\hline $\begin{array}{c}\text { Sr. } \\
\text { No. }\end{array}$ & $\begin{array}{c}\text { Bacterial } \\
\text { Culture }\end{array}$ & $\begin{array}{c}\mathbf{( \mathbf { m a x } -} \\
\mathbf{n m})\end{array}$ & DOP & $\begin{array}{c}\text { Biomass } \\
(\mathbf{g} / \mathbf{1 0 0 m l})\end{array}$ & \% RSA & $\begin{array}{c}\text { Chemical } \\
\text { nature }\end{array}$ & Predicted pigment \\
\hline $\mathbf{1}$ & SL2 & $\mathbf{4 7 3}$ & $\mathbf{2 . 8 1}$ & $\mathbf{2 . 8 9}$ & $\mathbf{5 3}$ & Carotenoid & Astaxanthin \\
\hline $\mathbf{2}$ & SL4-1 & 450 & 1.6 & 3.28 & 19 & Carotenoid & $\beta$-Carotene \\
\hline $\mathbf{3}$ & SL10 & 473 & 3.4 & 1.73 & 21 & Carotenoid & Astaxanthin \\
\hline $\mathbf{4}$ & SL4* & 463 & 1.1 & 2.62 & 60 & Non- carotenoid & -- \\
\hline $\mathbf{5}$ & SL2y & 437 & 0.6 & 2.84 & 11 & Non- carotenoid & -- \\
\hline
\end{tabular}

Table.5b Quaternary screening of carotenoid producing bacteria isolated from Marine water

\begin{tabular}{|c|c|c|c|c|c|c|c|}
\hline $\begin{array}{c}\text { Sr. } \\
\text { No. }\end{array}$ & $\begin{array}{c}\text { Bacterial } \\
\text { Culture }\end{array}$ & $\begin{array}{c}(\mathbf{\lambda m a x}- \\
\mathbf{n m})\end{array}$ & DOP & $\begin{array}{c}\text { Biomass } \\
\mathbf{( g / 1 0 0 m l})\end{array}$ & $\begin{array}{c}\mathbf{\%} \\
\text { RSA }\end{array}$ & $\begin{array}{c}\text { Chemical } \\
\text { nature }\end{array}$ & $\begin{array}{c}\text { Predicted } \\
\text { Pigment }\end{array}$ \\
\hline $\mathbf{1}$ & DKMIII & $\mathbf{4 5 0}$ & $\mathbf{1 . 0 0}$ & $\mathbf{0 . 2 3 7}$ & $\mathbf{2 3}$ & Carotenoid & 及-Carotene \\
\hline $\mathbf{2}$ & DABW1 & 463 & 0.66 & 0.234 & 14 & Carotenoid & Cryptoxanthin \\
\hline $\mathbf{3}$ & DGM & 436 & 0.41 & 0.232 & 12 & Non-Carotenoid & -- \\
\hline
\end{tabular}

Table.5c Quaternary screening of carotenoid producing bacteria isolated from Aurangabad and Hingoli samples

\begin{tabular}{|c|c|c|c|c|c|c|c|}
\hline $\begin{array}{c}\text { Sr. } \\
\text { No. }\end{array}$ & $\begin{array}{c}\text { Bacterial } \\
\text { Culture }\end{array}$ & $(\boldsymbol{\lambda m a x}-\mathbf{n m})$ & DOP & $\begin{array}{c}\text { Biomass } \\
(\mathbf{g} / \mathbf{1 0 0 m})\end{array}$ & $\begin{array}{c}\boldsymbol{\%} \\
\mathbf{R S A}\end{array}$ & $\begin{array}{c}\text { Chemical } \\
\text { nature }\end{array}$ & $\begin{array}{c}\text { Predicted } \\
\text { Pigment }\end{array}$ \\
\hline $\mathbf{1}$ & SSD2 & 468 & 0.93 & 0.347 & 18 & Carotenoid & Cryptoxanthin \\
\hline $\mathbf{2}$ & SWT3 & 536 & 2.69 & 0.921 & 56 & Non-Carotenoid & Prodigiosin \\
\hline $\mathbf{3}$ & SS1 & $\mathbf{4 7 4}$ & $\mathbf{1 . 4 2}$ & $\mathbf{0 . 5 3 2}$ & $\mathbf{2 3}$ & Carotenoid & Astaxanthin \\
\hline
\end{tabular}


Table.6 Munsell color code of the pigment produced by screened isolate

\begin{tabular}{|c|c|c|c|c|}
\hline $\begin{array}{l}\text { Sr. } \\
\text { No. }\end{array}$ & Isolate & Systematic Name & Munsell color code & $\begin{array}{l}\text { Appearance of } \\
\text { pigment }\end{array}$ \\
\hline 1 & SL2 & Vivid yellow Red & 1.3YR6.6/17.5 & [ata \\
\hline 2 & SL4* & Vivid yellow red & 4.0YR 6.7/16.9 & एक्ष \\
\hline 3 & SL4-1 & Vivid reddish yellow & $1.0 \mathrm{Y} 7.9 / 13.4$ & aba \\
\hline 4 & SL10 & Vivid yellow Red & 4.0YR 6.7/1 & एक \\
\hline 5 & SL2y & Vivid Greenish yellow & 7.1Y8.8/15.0 & tas \\
\hline 6 & DKMIII & Vivid reddish yellow & $1.0 Y 7.9 / 13.4$ & Fota \\
\hline 7 & DABW1 & Vivid reddish yellow & 4.0YR 6.7/16.9 & एवa \\
\hline 8 & DGM & Vivid Greenish Yellow & $1.0 Y 8.8 / 15.0$ & Exas \\
\hline 9 & SSD2 & Light Yellow Red & 1.5YR 7.0/10.7 & Exa \\
\hline 10 & SWT3 & Deep yellow Red & 9.0R 4.5/12.3 & Ear \\
\hline 11 & SS1 & Vivid yellow Red & 1.3YR6.6/17.5 & Eog \\
\hline
\end{tabular}

Figure.1a Distribution of number of samples collected according to source of sample,1b. distribution of samples according to location of samples collected

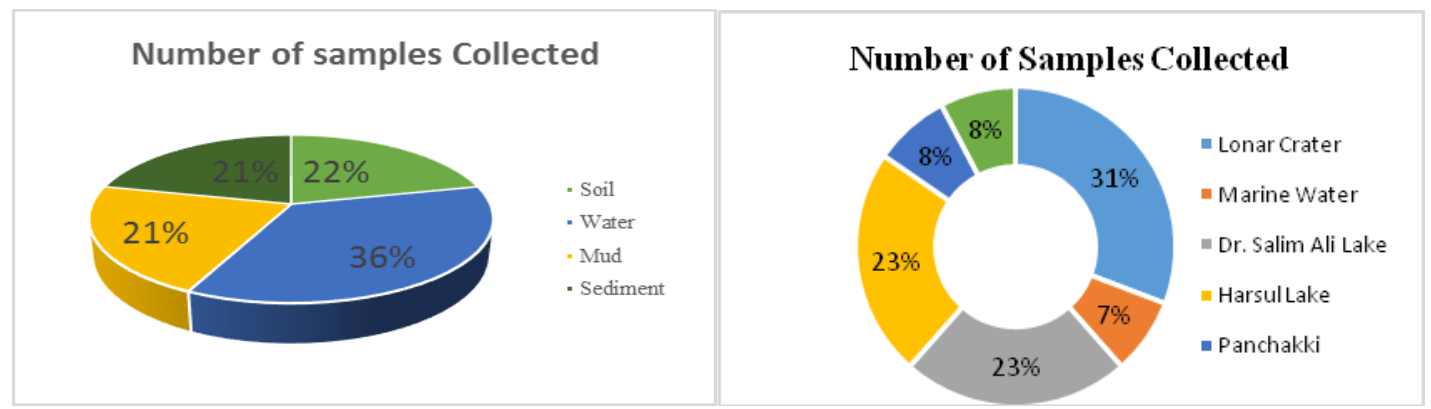

Figure.2 2a. Distribution of bacterial isolates from different sources of isolation, $\mathbf{2 b}$. Distribution of primarily screened isolates according to pigment produced

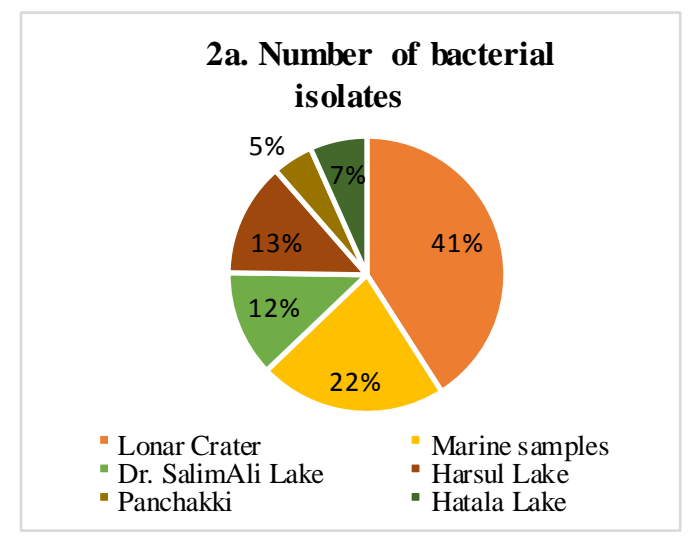

\section{2b. Pigmented Bacterial Isolates}

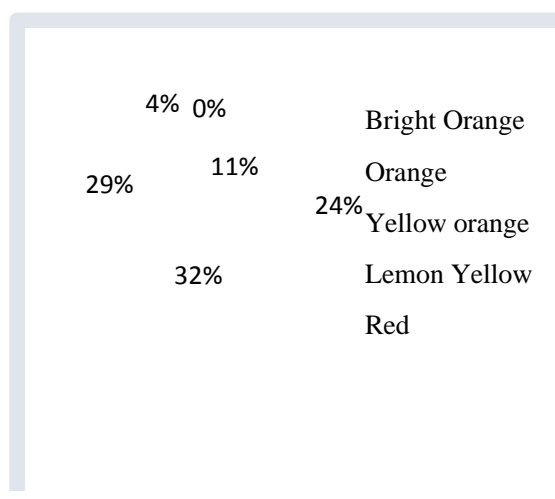


Secondary Screening of carotenoid producing Bacteria

Secondary screening was done based on qualitative $\mathrm{pH}$ and $\mathrm{NaCl}$ tolerance of 105 pigmented bacteria. All isolates were grown on nutrient agar with varying $\mathrm{pH}(6-12)$ and $\mathrm{NaCl}(1-6 \%)$ separately where, out of all primarily screened isolates 12 pigmented isolates from Lonar Crater, 12 pigmented isolates from marine samples and 07 from fresh water lakes isolates were screened secondarily based on bright pigmentation with broad range of $\mathrm{pH}$ (6-12) and salt (1-6\%) tolerance). Most of secondarily screened isolates shown growth above $\mathrm{pH} 11.0$ and 4\% $\mathrm{NaCl}$ with reduced pigmentation while some isolates do not grow at high $\mathrm{pH}$ and concentration of $\mathrm{NaCl}$, such isolates were eliminated for further studies during secondary screening(Table-3a, $3 \mathrm{~b}$ and 3c). Similar studies were carried out by Lee $e t$ al., (2004) where, astaxanthin producing Paracoccus haeundaensis sp. nov. showed $\mathrm{pH}$ tolerance in the range 6-10.5 and $\mathrm{NaCl}$ tolerance up to $6 \%$. Zheng et al., (2011) found that Paracoccus beibuensis $\mathrm{JLT}_{12} 24^{\mathrm{T}}$ can grow between $\mathrm{pH} 6.0$ to 8.0 and $\mathrm{NaCl}$ i.e. 2to $15 \%$. In these research studies, emphasis was given to screen potential carotenoid producing bacterial isolate and hence, screening was done based on both $\mathrm{pH}$ and $\mathrm{NaCl}$ tolerance. None of similar research studies were found where, combined criteria of tolerance to $\mathrm{pH}$ and $\mathrm{NaCl}$ used for screening of carotenoid producing bacteria which highlights the uniqueness of the current studies.

\section{Tertiary Screening of carotenoid producing Bacteria}

Total 31 pigmented isolates showing broad range $\mathrm{pH}$ between 6-12 and $\mathrm{NaCl}$ up to 1-6\% tolerance was subjected for tertiary screening based on extraction of pigment and its spectrophotometric characterization. According to the spectrophotometric analysis, $\lambda_{\max }$ of extracted pigments from different isolates were different varying between 400 $600 \mathrm{~nm}$ (Table $-4 \mathrm{a}, 4 \mathrm{~b}$, and 4c). The degree of pigmentation is a ratio of biomass to pigment produced and it is considered as efficiency of bacteria to produce carotenoids. Based on spectrophotometric characters and degree of pigmentation 05 isolates from Lonar crater, 03 from marine water samples and 03 isolates from fresh water lakes were selected for quaternary screening. The selected isolates showed pigments which are predicted based on their absorption maxima as SL2 as astaxanthin (473 nm), SL4-1 as zeaxanthin (443 nm), SL10 as astaxanthin (475 nm), $\mathrm{SL}^{*}$ as $\beta$-carotene (449 $\mathrm{nm}$ ), DKMIII as $\beta$ carotene $(450 \mathrm{~nm})$, DABW1 as cryptoxanthin $(463 \mathrm{~nm})$ and DGM as cryptoxanthin (436 $\mathrm{nm})$. The Munsell color code of each pigment from screened bacteria was determined by using Munsell color android application (Table-6).

El-Banna et al., (2012) described pigmented yeast colony color with the help of Munsell color chart as 10R7/8. Rodriguez-Amaya et al., (1999) suggested that absorption spectra of $\beta$-carotene are to be around $450 \mathrm{~nm}$. Shatila et al., (2013) analyzed orange pigment from Exiguobacterium within wavelength region 400-690 $\mathrm{nm}$ and demonstrated shoulder peak at $463 \mathrm{~nm}$ which is a typical pattern of absorption spectrum of carotenoids. Oren et al., (2001) extracted red carotenoid pigment from halophilic bacteria Salinibacter and analyzed it spectrophotometrically and found $482 \mathrm{~nm}$ as absorption maxima with a shoulder at about $510 \mathrm{~nm}$. In this research, for tertiary screening extraction and analysis of pigment was done to know the type of carotenoid, pigment production potential of isolate and possible applications were also considered. Very few research studies were found where spectrophotometric characterization and degree of pigmentation was evaluated as screening strategy. 
Quaternary screening based on antioxidant activity performed by DPPH assay

The isolates showing good degree of pigmentation and characteristic $\lambda_{\max }$ of carotenoid pigments were selected for evaluation of its antioxidant potential where, crude methanolic extract from 11 tertiary screened isolates were evaluated for antioxidant activity or free radical scavenging activity (\% RSA) by DPPH method. The stable radical of DPPH is used frequently for evaluation of antioxidant activity of natural colorant product. Out of 11 selected isolates SL2, DKMIII and SS1 showed highest i.e. $53 \%, 23 \%$ and $23 \%$ free radical scavenging activity respectively. The isolate SL2 showed good degree of pigmentation and \% RSA i.e. 2.81 and 53\% (Table-5a). Sasidharan et al., (2013) also used degree of pigmentation of carotenoid producing bacteria as a screening criterion where, highest degree of pigmentation was shown by bacterial isolate RS7 i.e. 8.31. \% RSA was evaluated by Arulselvi et al., (2014) for yellow pigment from bacterial strain using DPPH method and observed $70 \%$ activity. Nishino et al., (2000) suggested that DPPH free radical scavenging activity of methanol extract depends on concentration of carotenoids. According to Sandesh (2007) generally carotenoid potential for antioxidants vary many times 'in vivo' due to pro-oxidant effect. In this research studies evaluation of \% RSA used as last criteria for selection of isolate which highlights importance of carotenoids as an antioxidant molecule. Antioxidant potential of selected isolate increases its applicability at industrial level for production of carotenoids.

Isolate SL2 was finally selected as it can produce bright orange pigment, tolerate wide range of $\mathrm{pH}$ between 6-12 and $\mathrm{NaCl}$ up to 6\% with good degree of pigmentation (2.81), methanolic extract of pigment showed absorption maxima at $473 \mathrm{~nm}$ with characteristic shoulder peak of ketocarotenoids and 53\% RSA and hence, it was finally screened, selected and preserved for further studies.

\section{References}

1. Jk,,Arulselvi Indra $P$, Umamaheshwari $S$, Ranandkumar Sharma G, Karthik C. and Jayakrishna C. (2014) Screening of yellow pigment Producing Bacterial Isolates from Various Eco-Climatic Areas and Analysis of the Carotenoid Produced by the Isolate. J. Food Process Technol. $5: 1$

2. Arunkumar K. Ramasamy and V. Udayasuriyan (2006) Isolation and characterization of a yellow pigmented colony forming bacterium for carotenogenesis. Biotechnology 5 (1):7982

3. Balraj Janani, KiruthikaPannerselvam and Angayarkanni Jayaraman. (2014) Isolation of Pigmented Marine Bacteria Exiguobacterium Sp. From Peninsular Region of India and A Study On Biological Activity of Purified Pigment. International Journal of Scientific and Technology Research. 375-384.

4. Bhatt R M., Thankamani M. Media optimization. (2015) Extraction and Partial Characterization of an Orange Pigment from Salinococcus sp. MKJ997975. Int. J. of Life sci. and Pharma Research. 4(2): 85-89.

5. Brown Seward R. (1963). Bacterial carotenoids from freshwater sediments. Limnol. Oceanog., 8: 352-353.

6. El-Banna El Rhman Amr Abd, Amal Mohamed Abd El- Razek, Rafik ElMahdy, (2012). Isolation, Identification and screening of Carotenoid producing Strains of Rhodotorula giltinis. Food and Nutri. Sci. 3: 627-633.

7. Godlinho A. and Bhosle S. (2008), Carotenes produced by alkalophilic 
orange-pigmented Microbacterium

strain of arborescens-AGBS isolated from costal sand dunes, Indian Journal of Marine Sciences. 37(3) 307312

8. Lee Jae Hyung, Yun Sook Kim, Tae- Jin Choi, Won Jae Lee and Young Tae Kim (2004). Paracoccus haeundaensis sp. nov., a Gram-negative halophilic, astaxanthin- producing bacterium. Int. J. of Syst. And Evol. Microbiol. 54: 16991702.

9. Nishino T, Shibahara-Sone H, KikuchiHayakawa H, Lshikawa F. (2000) Transit of radical scavenging activity of milk products prepared by Millard reaction and Lactobacillus casei strain shirota fermentation through the Hamster intestine. J Dairy Sci. 83:915-922.

10. Oren Aharon and Francisco RodriguezValera, (2001). The contribution of Halophilic bacteria to red coloration of saltern crystallizer ponds. FEMS microbiology ecology 36: 123-130.

11. Pathak Anupama P. and Aparna G. Sardar, (2012). Isolation and characterization of carotenoid producing Haloarchaea from solar saltern of Mulund, Mumbai, India. Ind. J. of Nat. Resour. (4): 483-488.
12. Rodriguez-Amaya, D.B., and Kimura, M. (2004). Harvest Plus Handbook for Carotenoid Analysis. Harvest Plus Technical Monograph Int. J. Curr. Microbiol. App. Sci 2(12): 176-191 Series2; IFPRI: Washington, DC, USA, and CIAT: Cali, Colombia.

13. Sasidharan P., Raja R., Karthik C., Ranandkumar Sharma, Indra Arulselvi P. (2013) Isolation and characterization of yellow pigment producing Exiguobacterium sps. J. Biochem. Tech. 4:632-635

14. Shatila F., Hoda Yusef and Hanafy Holail (2013) Pigment production by Exiguobacterium aurantiacum $\mathrm{FH}$, a novel Labanese strain. Int. J. Curr. Microbiol. App. Sci. 2(12), pp: 176-191.

15. Snandesh K. (2007) Biotechnoligical production of micro-algal carotenoids with reference to Astaxanthin and evaluation of its biological activity. Online Thesis.

16. Zeni J., R. Colet, K. Cence, L. Tiggemann, G. Toniazzo, R.L. Cansian, M. Di Luccio, D. Oliveira and E. Valduga, (2011). Screening of microorganisms for production of carotenoids. J. of Food 9(2): 160-166.

\section{How to cite this article:}

Deepti D. Dhere, S. M. Dharmadhikari and Pandhare, R. S. 2020. Isolation and Screening of Carotenoid Producing Bacteria. Int.J.Curr.Microbiol.App.Sci. 9(01): 941-952. doi: https://doi.org/10.20546/ijcmas.2020.901.106 